\title{
Algorithms using genome-wide association studies for prediction of effectiveness of biologics in rheumatoid arthritis
}

\author{
Marowa Hashimoto $^{12 *}$, Keiko Funahashi ${ }^{12)}$, Toshihisa Maeda ${ }^{2)}$, Akira Sagawa ${ }^{3)}$, \\ Tomomaro Izumihara ${ }^{4}$, Eisuke Shono5), Hiroaki Matsuno ${ }^{6}$, Koji Fukuda", Shinya Hayashi", \\ Ryosuke Kuroda", Tsukasa Matsubara ${ }^{12)}$ \\ 1) Research Institute of Joint Diseases, Kobe, Japan \\ 2) Matsubara Mayflower Hospital, Kato, Japan \\ 3) Sagawa Akira Rheumatology Clinic, Sapporo, Japan \\ 4) Izumihara Rheumatic and Medical Clinic, Kagoshima, Japan \\ 5) Shono Rheumatology Clinic, Fukuoka, Japan \\ 6) Matsuno Clinic for Rheumatic Disease, Toyama, Japan \\ 7) Department of Orthopaedic Surgery, Kobe University Graduate School of Medicine, Kobe, Japan
}

\begin{abstract}
:
Purpose: Two anti-TNF biologics, infliximab and etanercept, are extremely useful for inflammatory diseases such as rheumatoid arthritis (RA). However, approximately 20 to 30\% of RA have been described as non-responders. Here, we analyzed the statistical relationships of whole genome single nucleotide polymorphisms (SNPs) with remission or low disease activity (LDA) among RA and developed algorithms using SNPs to predict effectiveness of these biologics.

Methods: The total study subjects (first, second and validation population for each infliximab and etanercept) were 260 RA patients for infliximab and another 251 RA patients for etanercept. Effectiveness of infliximab and etanercept was assessed using DAS28-CRP. In first, second and the combined population, relationships of 277,339 SNPs with remission and LDA were analyzed using case-control analyses by Fisher's exact tests for each infliximab and etanercept. We picked up SNPs $(P<0.05)$ from each first, second and combined population. Then, 10 SNPs with lower $P$ value in the combined population were selected from common SNPs among the first, second and combined populations. We developed algorithms using the 10 SNPs to predict effectiveness of infliximab and etanercept. In the validation population, availability of the algorithms was evaluated.
\end{abstract}

Results: Using combined infliximab-remission and LDA algorithms in the validation population, the accuracy was 90.4\%. Using combined etanercept-remission and LDA algorithms in validation population, the accuracy was $76.8 \%$.

Conclusions: The combined use of our two algorithms using SNPs may be very useful in the prediction of remission or LDA before treatment with infliximab or etanercept.

\section{Keywords:}

infliximab, etanercept, effectiveness, single nucleotide polymorphism, GWAS

\section{Introduction}

Rheumatoid arthritis (RA) is a progressive autoimmune disease well defined by widely accepted symptoms such as chronic joint inflammation and structural damage [1]. In $\mathrm{RA}$, the first purpose of treatment is to control symptoms

\footnotetext{
*Corresponding author: Marowa Hashimoto, mhashimoto@leaf.ocn.ne.jp

Article history: Received 22 August 2019, Received in revised form 18 December 2019, Accepted 27 December 2019

https://doi.org/10.46459/pmu.2019019

Copyright (C) 2020 International Society of Personalized Medicine
} 
and avoid joint damage. Predicting the prognosis of RA is very important in order to manage the disease. However, its clinical features and the molecular pathways are heterogeneous [2,3]. Therefore, the responses to treatments vary among RA patients, which make it difficult to predict RA patient's responsiveness to drugs.

In treatment for RA at present, using biological agents including tumor necrosis factor (TNF) blockades is extremely useful because these agents specifically inhibit immune responses and inflammation. Achievement of remission or low disease activity (LDA) in biological treatments for RA is currently one of the most important therapeutic targets. The importance of remission or LDA as outcomes has been highlighted by European League Against Rheumatism (EULAR) recommendations [4]. However, several studies have reported that approximately 20 to $30 \%$ of RA patients treated with these agents experience only transient clinical improvement or have no benefit because of individual differences such as lifestyles, past history and genetic factors among the patients $[5,6]$. Therefore, predicting the effectiveness of biologics is not easy before treatment. So far, several studies have reported that many single nucleotide polymorphisms (SNPs) affect biological efficasy [7-9]. These studies, however, only focused on SNPs located on genes associated with the TNF cascade, potentially missing other SNPs affecting efficacy. It has also been reported that some genes expression profiles in blood could predict the response to a biological agent [10]. However, using SNPs has not thus far been reported.

In this study, in order to develop algorithms using SNPs to predict effectiveness of infliximab (IFX) and etanercept (ETN) before their first administration, we analyzed the statistical relationships of whole genome SNPs with remission or LDA among IFX- or ETN-treated RA patients. Then, we examined whether the algorithms could predict the effectiveness of these biologics.

\section{Material and methods}

\subsection{Study subjects}

We recruited 511 RA patients receiving treatment of IFX or ETN from 6 hospitals (A to F hospital). The patients were divided into three populations by IFX or ETN treatment. The first population for IFX treatment included 94 RA patients recruited from hospital A. The second population for IFX treatment included 92 RA patients recruited from hospitals B, C, D, E or F. The validation population for IFX treatment included 72 RA patients recruited from all hospitals. The first population for ETN treatment included 88 RA patients recruited from hospital A. The second population for ETN treatment included $81 \mathrm{RA}$ patients recruited from hospitals B, C, D, E or F. The validation population for ETN treatment included 73 RA patients recruited from all hospitals. Written informed consent to participate in this study was obtained from each patient. This study was ap- proved by the ethical committee for analytical research on the human genome of the Matsubara Mayflower Hospital.

\subsection{Assessment of disease activity}

The Disease Activity Score with 28 joints using Creactive protein (DAS28-CRP) was used in order to assess efficacy of IFX or ETN for each patient. Remission and LDA criteria were determined by DAS28-CRP score within 24 to 30 weeks after the first administration of IFX or ETN to each patient. Patients were categorized by their DAS28CRP score into two groups: remission (DAS28-CRP score $<2.3$ ) and non-remission (DAS28-CRP score $\geq 2.3$ ). Patients were also categorized by their DAS28-CRP score into two groups: LDA including remission (DAS28-CRP score $<2.7$ ) and non-LDA (DAS28-CRP score $\geq 2.7$ ).

\subsection{Genome-wide SNP genotyping}

The patients' whole blood samples were used for DNA extraction at Mitsubishi BCL Inc. Genome wide SNP genotyping were performed at deCode genetics Inc. (Reykjavik, Iceland) using Illumina HumanHap300K chip technology (Illumina Corp., San Diego, CA, USA). After genotyping, 277,339 of 317,503 SNPs excluded SNPs with call rates $<90 \%$ and minor allele frequency $<1 \%$ were used in the case-control analysis described below.

\subsection{Statistical analysis}

We used the case-control analyses to analyze the relationships of 277,339 SNPs with remission/non-remission and LDA/non-LDA by Fisher's exact tests using Plink program (version 1.07). The case-control analyses were carried out in each first population, second population and the population combined first and second population.

\subsection{Developing algorithms}

After case-control analyses, we picked up SNPs ( $P$ $<0.05)$ that tended to be associated with remission/nonremission from each first, second and combined populations for IFX. The 10 SNPs with lower $P$ value in combined the population were selected from common SNPs $(P<0.05)$ among first, second and combined populations. We scored each of the 10 SNPs in order to develop algorithms for prediction of IFX remission or non-remission. We gave a score of +1 to major allele homo in remission, score of 0 to hetero and score of -1 to major allele homo in non-remission. The total of 10 SNPs score was used in order to predict remission or non-remission. The optimum cut-off value was determined based on receive operating characteristic (ROC) analysis. Then, in order to evaluate functionality of this algorithm, we calculated accuracy, specificity and sensitivity of this algorithm in the validation population. The accuracy, specificity and sensitivity were calculated using formulas: (true positive + true negative)/total, true negative/(false positive + true negative) and true positive/(true positive + false negative), respectively. Similarly, algorithms for prediction of IFX LDA/non-LDA, ETN remission/non-remission and 
Table 1. Clinical characteristics of patients

\begin{tabular}{|c|c|c|c|c|c|c|}
\hline \multirow[b]{2}{*}{ Population } & \multicolumn{3}{|c|}{ infliximab $(\mathrm{n}=260)$} & \multicolumn{3}{|c|}{ etanercept $(n=251)$} \\
\hline & First & Second & Validation & First & Second & Validation \\
\hline Number of patients & 95 & 92 & 73 & 88 & 81 & 82 \\
\hline Age (years) & $58.5 \pm 12.7$ & $53.1 \pm 13.1$ & $56.0 \pm 11.4$ & $61.6 \pm 11.7$ & $53.7 \pm 13.1$ & $55.1 \pm 15.1$ \\
\hline Female $(\%)$ & 73.7 & 89.1 & 82.2 & 75.0 & 93.8 & 80.5 \\
\hline Disease duration (years) & $9.8 \pm 8.8$ & $10.5 \pm 9.1$ & $10.2 \pm 10.1$ & $11.2 \pm 10.2$ & $11.3 \pm 8.7$ & $8.0 \pm 8.5$ \\
\hline DAS28 (CRP) & $5.0 \pm 1.3$ & $4.8 \pm 1.2$ & $4.9 \pm 1.2$ & $5.0 \pm 1.2$ & $4.7 \pm 1.1$ & $4.8 \pm 1.4$ \\
\hline $\operatorname{high}(>5.1)$ & 49 & 38 & 30 & 48 & 27 & 34 \\
\hline moderate + low $(\leq 5.1)$ & 46 & 54 & 43 & 40 & 54 & 48 \\
\hline \multicolumn{7}{|c|}{ Steinbrocker's radiographic stage (disease stage) } \\
\hline I (Early) + II (Moderate) & 20 & 34 & 32 & 16 & 23 & 13 \\
\hline III (Severe) + IV (Terminal) & 72 & 58 & 25 & 70 & 57 & 38 \\
\hline unknown & 3 & - & - & 2 & 1 & 31 \\
\hline \multicolumn{7}{|c|}{ Steinbrocker's functional class (degree of dysfunction) } \\
\hline $\mathrm{I}+\mathrm{II}$ & 75 & 72 & 53 & 73 & 64 & 45 \\
\hline $\mathrm{III}+\mathrm{IV}$ & 17 & 20 & 4 & 13 & 16 & 8 \\
\hline unknown & 3 & - & - & 2 & 1 & 29 \\
\hline \multicolumn{7}{|l|}{ Remission or non-remission } \\
\hline remission & 22 & 17 & 14 & 30 & 24 & 37 \\
\hline non-remission & 72 & 75 & 59 & 58 & 57 & 45 \\
\hline \multicolumn{7}{|c|}{ Low disease activity or non-low disease activity } \\
\hline low disease activity & 29 & 30 & 22 & 44 & 38 & 47 \\
\hline non-low disease activity & 66 & 62 & 51 & 44 & 43 & 35 \\
\hline
\end{tabular}

Values are mean $\pm \mathrm{SD}$, number of the patients

class I: Can perform all activities, class II: Moderate restriction of activities, class III: Marked restriction of activities, class IV: Incapacitated

Table 2. Selected 10 SNPs associated with infliximab remission

\begin{tabular}{lllllllll}
\hline SNP & Chr & A1 & F_R & F_N & A2 & Combined $P$ & OR & Gene \\
\hline rs10860738 & 12 & A & 0.6154 & 0.3265 & G & $6.4 \times 10^{-6}$ & 3.3 & - \\
rs2181276 & 1 & C & 0.2692 & 0.5276 & T & $6.3 \times 10^{-5}$ & 0.33 & HIVEP3 \\
rs751511 & 13 & T & 0.6538 & 0.398 & C & $6.5 \times 10^{-5}$ & 2.9 & - \\
rs7603262 & 2 & A & 0.3077 & 0.5646 & C & $6.7 \times 10^{-5}$ & 0.34 & AP1S3 \\
rs763713 & 2 & C & 0.5385 & 0.2959 & A & $9.5 \times 10^{-5}$ & 2.8 & PAIP2B \\
rs4629018 & 17 & A & 0.6667 & 0.4184 & G & $1.2 \times 10^{-4}$ & 2.8 & JUP \\
rs7623638 & 3 & G & 0.5769 & 0.3367 & A & $1.4 \times 10^{-4}$ & 2.7 & EPHA6 \\
rs6598193 & 12 & G & 0.6154 & 0.3707 & A & $1.6 \times 10^{-4}$ & 2.7 & - \\
rs2461224 & 10 & G & 0.2821 & 0.5238 & A & $1.9 \times 10^{-4}$ & 0.36 & TACC2 \\
rs12878216 & 14 & A & 0.5641 & 0.3265 & G & $2.0 \times 10^{-4}$ & 2.7 & TRA \\
\hline
\end{tabular}

Chr: chromosome number, A1: allele 1, F_R: frequency of allele 1 in remission group,

F_N: frequency of allele 1 in non-remission group, A2: allele 2,

Combined $P: P$ value in the population combined first and second population, OR: odds ratio,

Gene: Genes mapped to the SNP (NCBI genome build 106)

ETN LDA/non-LDA were developed and evaluated. We also evaluated functionality of algorithms for combined remission and LDA algorithm.

\section{Results}

The basic characteristics of the patients according to each population are presented in Table 1. The total patients of populations for IFX were 260 patients. The total patients of populations for ETN were 251 patients. The first population for IFX was 95 patients aged $58.5 \pm 12.7$ years (mean \pm
SD), the second population for IFX was 92 patients aged $53.1 \pm 13.1$ and the validation population for IFX was 73 patients aged $56.0 \pm 11.4$. The first population for ETN was 88 patients aged $61.6 \pm 11.7$, the second population for ETN was 81 patients aged $53.7 \pm 13.1$ and the validation population for ETN was 82 patients aged $55.1 \pm 15.1$.

With regard to IFX remission, 1,328 SNPs were chosen. The $P$ value for 314 SNPs was less than 0.001 , and for 1014 SNPs was less than 0.01 . Table 2 presents the selected 10 SNPs which were strongly associated with IFX remission. Odds ratio for rs 10860738 was $3.3\left(P=6.4 \times 10^{-6}\right)$, rs 
Table 3. Selected 10 SNPs associated with infliximab low disease activity

\begin{tabular}{lllllllll}
\hline SNP & Chr & A1 & F_L & F_N & A2 & Combined $P$ & OR & Gene \\
\hline rs10848816 & 12 & G & 0.65 & 0.2922 & A & $5.5 \times 10^{-6}$ & 4.5 & TSPAN9 \\
rs2288947 & 19 & G & 0.6 & 0.259 & A & $8.0 \times 10^{-6}$ & 4.3 & TINCR \\
rs1933437 & 13 & C & 0.55 & 0.2259 & T & $1.0 \times 10^{-5}$ & 4.2 & FLT3 \\
rs7715337 & 5 & A & 0.4 & 0.1325 & G & $1.4 \times 10^{-5}$ & 4.4 & LOC101927421 \\
rs9486755 & 6 & T & 0.425 & 0.1506 & C & $2.0 \times 10^{-5}$ & 4.2 & SEC63 \\
rs7513602 & 1 & A & 0.075 & 0.4187 & G & $2.4 \times 10^{-5}$ & 0.11 & KIF26B \\
rs1941238 & 18 & G & 0.45 & 0.1717 & A & $3.4 \times 10^{-5}$ & 3.9 & - \\
rs7866925 & 9 & T & 0.35 & 0.1114 & C & $3.4 \times 10^{-5}$ & 4.3 & AKNA \\
rs4128811 & 4 & G & 0.8 & 0.4548 & A & $3.7 \times 10^{-5}$ & 4.8 & - \\
rs4078293 & 10 & C & 0.625 & 0.3 & T & $3.9 \times 10^{-5}$ & 3.9 & - \\
\hline
\end{tabular}

Chr: chromosome number, A1: allele 1, F_R: frequency of allele 1 in low disease activity group, F_N: frequency of allele 1 in non-low disease activity group, A2: allele 2,

Combined $P: P$ value in the population combined first and second population, OR: odds ratio, Gene: Genes mapped to the SNP (NCBI genome build 106)

Table 4. Selected 10 SNPs associated with etanercept remission

\begin{tabular}{lllllllll}
\hline SNP & Chr & A1 & F_R & F_N & A2 & Combined $P$ & OR & Gene \\
\hline rs10872224 & 6 & G & 0.1852 & 0.4304 & T & $9.2 \times 10^{-6}$ & 0.30 & - \\
rs10484867 & 6 & A & 0.1852 & 0.4204 & G & $2.4 \times 10^{-5}$ & 0.31 & - \\
rs9375195 & 6 & A & 0.2407 & 0.4739 & G & $4.6 \times 10^{-5}$ & 0.35 & - \\
rs6932561 & 6 & T & 0.5943 & 0.3522 & C & $4.9 \times 10^{-5}$ & 2.7 & - \\
rs2281449 & 6 & G & 0.6481 & 0.413 & A & $6.8 \times 10^{-5}$ & 2.6 & TNFRSF21 \\
rs2175491 & 15 & T & 0.4907 & 0.2652 & C & $7.0 \times 10^{-5}$ & 2.7 & UNC13C \\
rs3212198 & 20 & T & 0.4907 & 0.2696 & C & $8.1 \times 10^{-5}$ & 2.6 & HNF4A \\
rs12612022 & 2 & C & 0.2037 & 0.4211 & T & $8.5 \times 10^{-5}$ & 0.35 & CNTNAP5 \\
rs12216178 & 6 & G & 0.1944 & 0.4087 & A & $1.2 \times 10^{-4}$ & 0.35 & - \\
rs11665898 & 19 & G & 0.4444 & 0.2391 & A & $2.2 \times 10^{-4}$ & 2.5 & - \\
\hline
\end{tabular}

Chr: chromosome number, A1: allele 1, F_R: frequency of allele 1 in remission group,

F_N: frequency of allele 1 in non-remission group, A2: allele 2,

Combined $P: P$ value in the population combined first and second population, OR: odds ratio,

Gene: Genes mapped to the SNP (NCBI genome build 106)

2181276 was $0.33\left(P=6.3 \times 10^{-5}\right)$, rs751511 was $2.9(P=$ $\left.6.5 \times 10^{-5}\right)$, rs7603262 was $0.34\left(P=6.7 \times 10^{-5}\right)$, rs763713 was $2.8\left(P=9.5 \times 10^{-5}\right)$, rs4629018 was $2.8(P=1.2 \times$ $\left.10^{-4}\right)$, rs7623638 was $2.7\left(P=1.4 \times 10^{-4}\right)$, rs6598193 was $2.7\left(P=1.6 \times 10^{-4}\right)$, rs2461224 was $0.36\left(P=1.9 \times 10^{-4}\right)$ and rs12878216 was $2.7\left(P=2.0 \times 10^{-4}\right)$ in the combined population. The cut-off value for prediction of IFX remission or non-remission was a score of 2 points (remission $\geq$ 2 points, non-remission $\leq 1$ points). In the validation population, accuracy, specificity and sensitivity of the algorithm based on this cut-off value were $66.7 \%, 53.8 \%$ and $69.5 \%$, respectively.

With regard to IFX LDA, 3,122 SNPs were chosen. The $P$ value for 419 SNPs was less than 0.001, and for 2703 SNPs was less than 0.01 . Table 3 presents the selected 10 SNPs which were strongly associated with IFX LDA. Odds ratio for rs10848816 was $4.5\left(P=5.5 \times 10^{-6}\right)$, rs 2288947 was $4.3\left(P=8.0 \times 10^{-6}\right), \operatorname{rs} 1933437$ was $4.2(P=1.0 \times$ $\left.10^{-5}\right)$, rs7715337 was $4.4\left(P=1.4 \times 10^{-5}\right)$, rs9486755 was $4.2\left(P=2.0 \times 10^{-5}\right)$, rs7513602 was $0.11\left(P=2.4 \times 10^{-5}\right)$, rs
1941238 was $3.9\left(P=3.4 \times 10^{-5}\right)$, rs7866925 was $4.3(P=$ $\left.3.4 \times 10^{-5}\right)$, rs4128811 was $4.8\left(P=3.7 \times 10^{-5}\right)$ and rs 4078293 was $3.9\left(P=3.9 \times 10^{-5}\right)$ in the combined population. The cut-off value for prediction of IFX LDA or nonLDA was a score of -1 points (LDA $\geq-1$ points, non-LDA $\leq-2$ points). In the validation population, accuracy, specificity and sensitivity of the algorithm based on this cut-off value were $82.2 \%, 12.5 \%$ and $90.8 \%$, respectively.

With regard to ETN remission, 1,653 SNPs were chosen. The $P$ value for 521 SNPs was less than 0.001 , and for 1132 SNPs was less than 0.01. Table 4 presents the selected 10 SNPs which were strongly associated with ETN remission. Odds ratio for rs10872224 was $0.30\left(P=9.2 \times 10^{-6}\right)$, rs10484867 was $0.31\left(P=2.4 \times 10^{-5}\right)$, rs9375195 was 0.35 $\left(P=4.6 \times 10^{-5}\right)$, rs6932561 was $2.7\left(P=4.9 \times 10^{-5}\right)$, rs 2281449 was $2.6\left(P=6.8 \times 10^{-5}\right)$, rs2175491 was $2.7(P=$ $\left.7.0 \times 10^{-5}\right), \mathrm{rs} 3212198$ was $2.6\left(P=8.1 \times 10^{-5}\right)$, rs 12612022 was $0.35\left(P=8.5 \times 10^{-5}\right)$, rs 12216178 was $0.35(P=1.2 \times$ $\left.10^{-4}\right)$ and rs11665898 was $2.5\left(P=2.2 \times 10^{-4}\right)$ in combined population. The cut-off value for prediction of ETN remis- 
Table 5. Selected 10 SNPs associated with etanercept low disease activity

\begin{tabular}{lllllllll}
\hline SNP & Chr & A1 & F_R & F_N & A2 & Combined $P$ & OR & Gene \\
\hline rs676134 & 11 & C & 0.5179 & 0.2057 & T & $1.1 \times 10^{-6}$ & 4.1 & BACE1 \\
rs1564326 & 15 & C & 0.6786 & 0.3617 & T & $1.1 \times 10^{-5}$ & 3.7 & - \\
rs711280 & 6 & C & 0.3571 & 0.1241 & T & $1.6 \times 10^{-5}$ & 3.9 & - \\
rs9320236 & 6 & G & 0.4286 & 0.1738 & T & $2.3 \times 10^{-5}$ & 3.6 & SCML4 \\
rs3790112 & 16 & T & 0.2321 & 0.539 & G & $2.7 \times 10^{-5}$ & 0.26 & GNAO1 \\
rs2199724 & 15 & A & 0.7143 & 0.4113 & C & $3.3 \times 10^{-5}$ & 3.6 & - \\
rs1503575 & 12 & A & 0.4821 & 0.2163 & G & $3.5 \times 10^{-5}$ & 3.4 & - \\
rs12196723 & 6 & C & 0.3571 & 0.1312 & T & $3.7 \times 10^{-5}$ & 3.7 & WDR27 \\
rs7310134 & 12 & A & 0.4464 & 0.1915 & G & $3.8 \times 10^{-5}$ & 3.4 & - \\
rs1451117 & 4 & T & 0.1429 & 0.4357 & C & $4.0 \times 10^{-5}$ & 0.22 & - \\
\hline
\end{tabular}

Chr: chromosome number, A1: allele 1, F_R: frequency of allele 1 in low disease activity group, F_N: frequency of allele 1 in non-low disease activity group, A2: allele 2,

Combined $P: P$ value in the population combined first and second population, OR: odds ratio,

Gene: Genes mapped to the SNP (NCBI genome build 106)

sion was a score of +2 points (remission $>+2$ points, nonremission $<+2$ points). In the validation population, accuracy, specificity and sensitivity of the algorithm based on this cut-off value were $56.1 \%, 43.2 \%$ and $66.7 \%$, respectively.

With regard to ETN LDA, the picked up SNPs were 2,695 SNPs. The $P$ value for 320 SNPs was less than 0.001 , and for 2375 SNPs was less than 0.01. Table 5 presents the selected 10 SNPs which were strongly associated with ETN LDA. Odds ratio for rs676134 was $4.1\left(P=1.1 \times 10^{-6}\right)$, rs 1564326 was $3.7\left(P=1.1 \times 10^{-5}\right)$, rs711280 was $3.9(P=$ $\left.1.6 \times 10^{-5}\right)$, rs9320236 was $3.6\left(P=2.3 \times 10^{-5}\right), \mathrm{rs} 3790112$ was $0.26\left(P=2.7 \times 10^{-5}\right)$, rs 2199724 was $3.6(P=3.3 \times$ $\left.10^{-5}\right)$, rs 1503575 was $3.4\left(P=3.5 \times 10^{-5}\right)$, rs12196723 was $3.7\left(P=3.7 \times 10^{-5}\right)$, rs7310134 was $3.4\left(P=3.8 \times 10^{-5}\right)$ and rs1451117 was $0.22\left(P=4.0 \times 10^{-5}\right)$ in the combined population. The cut-off value for prediction of ETN LDA was a score of -2 points (LDA $>-2$ points, non-LDA $<-2$ points). In the validation population, accuracy, specificity and sensitivity of the algorithm based on this cut-off value were $73.2 \%, 50.0 \%$ and $76.4 \%$, respectively.

Using algorithm combined IFX-remission and LDA algorithms in the validation population, accuracy, specificity and sensitivity were $90.4 \%, 57.1 \%$ and $98.3 \%$, respectively. Using combined ETN-remission and LDA algorithms in the validation population, accuracy, specificity and sensitivity were $76.8 \%, 62.2 \%$ and $88.9 \%$, respectively.

\section{Discussion}

To our knowledge, our study is the first to develop algorithms to predict effectiveness of biologics such as IFX and ETN using only the results of genome-wide association studies (GWAS) among Japanese RA patients.

It has been reported that the SNPs in genes such as $\mathrm{Hu}-$ man Leukocyte Antigen-DRB 1 (HLA-DRB1) [7], Interleukin-10 (IL-10) [11], Interleukin-1 receptor antagonist (IL-1RN) [12] and Transforming Growth Factor- $\beta 1$ (TGF $\beta$
1) [13] affects the effectiveness of IFX or ETN. However, these studies have shown the relationships of only a few SNPs in genes involved in the TNF cascade with remission, and LDA in IFX and ETN treatment. Therefore, it is likely that SNPs in unknown genes associated with drug responsiveness were overlooked. In this regard, GWAS is powerful tool to collectively identify SNPs associated with drug response [14]. By using GWAS, it has been clear that three genomic regions showed significant or borderline significant associations with anti-TNF response in Japanese RA patients [15]. Moreover, as a predictor of response to ETN therapy in RA, GWAS identified CD84 [16]. Thus, conducting GWAS is valuable in identifying SNPs associated with the effectiveness of IFX or ETN. So far, it has not been established that SNPs can be used as prediction method for responsiveness of these biologics. In this study, we developed the algorithms using only SNPs based on the result of GWAS.

In our study, 10 SNPs were used for each algorithm. In case SNPs are used to predict the effectiveness of biologics, it seems that using more SNPs raises the accuracy of predicting the effectiveness. On the other hand, using more SNPs is difficult because of problems such as high cost and time required for analyzing. In addition, using more SNPs makes the algorithms for prediction complicated. However, algorithms using fewer SNPs probably show low accuracy of prediction. We, thus, selected 10 SNPs with lower $P$ value and developed the algorithms.

A previous study revealed that an eight-gene blood expression profile could predict the response to IFX in RA with an $85.7 \%$ prediction accuracy [10]. Another previous study also revealed that a comprehensive transcriptome analysis of white blood cells was capable of predicting efficacy of IFX for RA patients [17]. These reports, however, were based on blood RNA expression levels. Because blood RNA expression level may vary day to day, the result of prediction for response to biologics may vary. We, thus, tested prediction methods of IFX or ETN responses using 
SNPs as the most basic non-variable biomarker. Our SNP algorithm for prediction of remission or LDA in IFX treatment showed higher accuracy $(90.4 \%)$ than the two previous reports. With regard to ETN, a previous study has reported that cytokine profiling by proteomic analysis may predict responsiveness of ETN in RA patients with an $84.4 \%$ accuracy [18]. Our SNP algorithm for prediction of remission or LDA in ETN treatment showed a little lower accuracy (76.8\%) than this previous study. Nevertheless, our SNP algorithm can predict the responsiveness more quickly and cheaply than the cytokine profiling by proteomic analysis. Furthermore, another previous study has reported that even diagnostic sensitivity and specificity of rheumatoid factor for rheumatoid arthritis were $69 \%$ and $85 \%$, respectively [19]. In same study, the diagnostic sensitivity and specificity of antibodies against cyclic citrullinated peptide (anti-CCP antibodies) were also $67 \%$ and $95 \%$, respectively [19]. Our algorithms have a high usefulness compared to these sensitivity or specificity. Thus, it seems that our SNP algorithm is quite useful.

Our genome-wide SNP association analysis found some SNP loci mapped on notable or near notable genes. Rs 12878216 associated with IFX remission in our study is located on the $\mathrm{T}$ cell receptor alpha locus. Because $\mathrm{T}$ cell receptor-mediated $\mathrm{T}$ cell activation was enhanced in the peripheral blood, this SNP might have some kind of influence on the $\mathrm{T}$ cell immune responses in IFX treatment [20]. Rs 2281449 associated with ETN remission in our study is located on the TNF receptor superfamily member 21 gene (TNFRSF21). It has been reported that TNFRSF21, also known as death receptor 6 (DR6), enhances B cell expansion, survival and humoral responses in mice [21]. TNFRSF 21 also has an important regulatory role in limiting $\mathrm{T}$ cell immune responses [22]. Furthermore, the expression of TNFRSF21 is induced by TNF-alpha through activation of NF-kappaB [23]. These studies have suggested that this rs 2281449 SNP might affect B cell and T cell immune responses in treatment of ETN used as TNF blockade. Rs 6932561 associated with ETN remission in our study is located on the 5'-flanking region of the $C D 83$ gene. The $C D$ 83 gene locus was associated with RA in the Japanese population [24]. In RA, soluble CD83 levels were higher in synovial fluid than in plasma [25]. Early RA patients also increased levels of soluble CD83 in plasma [26]. However, the level of soluble CD83 in plasma did not correlate with RA activity [26]. In addition, the release of soluble CD83 was independent of TNF-alpha [26]. Therefore, relationship of CD83 with RA should be further examined in future research. Rs11665898 associated with ETN remission in our study located on the 5'-flanking region of the CD97 gene. In rheumatoid synovial tissues, CD97 and its ligand CD55 expression are expressed at high levels, and the CD97/CD55 pair may be of primary importance in maintaining and amplifying synovial inflammation [27]. This SNP located on the 5'-flanking region might affect the responsiveness of ETN through affecting CD97 expression and rheumatoid synovial inflammation.

Interestingly, there were no SNPs overlapping between IFX and ETN remission or LDA. This might be caused by the structural difference between IFX and ETN. IFX is a chimeric antibody against TNF-alpha, whereas ETN is a soluble form of extracellular region of TNF receptor.

Our algorithms have a limitation. These are mainly for Japanese RA patients because this is based on the results of GWAS among Japanese RA patients. It is well known that allele frequencies of most SNPs vary in different ethnic groups. Allele frequencies of SNPs we selected for our algorithms also varied compared with the allele frequencies of other ethnic groups reported in the HapMap database (http s://www.ncbi.nlm.nih.gov/snp). Therefore, our algorithms may not be applicable to non-Japanese RA patients. It is also well known that allele frequency of SNPs such as rs671 in aldehyde dehydrogenase 2 and rs1229984 in alcohol dehydrogenase 1B associated with alcohol sensitivity and drinking behavior vary from place to place within Japan $[28,29]$. Therefore, there is possibility that the SNPs we selected for our algorithms have area-specific allele frequency. However, our study RA patients were recruited from several regions in Japan. Thus, our algorithms seems to be applicable to most Japanese RA patients.

\section{Conclusions}

This is the first report of algorithms to predict the effectiveness of IFX or ETN using 10 SNPs selected from the results of genome wide SNP association analysis. Our SNP algorithms are very easy to use, and the algorithms combined IFX- or ETN-remission and LDA algorithms showed high accuracy (90.4\% and $76.8 \%$, respectively). Therefore, our algorithms may be very useful in the prediction of remission or LDA before treatment of IFX or ETN. Furthermore, this scoring method using SNPs may contribute to tailor-made treatments with biologics in the future.

\section{Conflicts of interest}

The authors have declared that no conflicts of interest exist.

\section{Acknowledgements}

We would like to thank Dr. Satoru Koyano for helping with the statistical analyses. We also would like to thank Ms. Carol Matsubara for proofreading the manuscript.

\section{References}

[1] Combe B. Early rheumatoid arthritis: strategies for prevention and management. Best Pract Res Clin Rheumatol 2007;21:27-42. doi: 10.1016/j.berh.2006.08.011.

[2] Arnett FC, Edworthy SM, Bloch DA, et al. The American Rheumatism Association 1987 revised criteria for the classification of rheumatoid arthritis. Arthritis Rheum 1988;31:315-24.. 
[3] Choy EHS, Panayi GS. Cytokine Pathways and Joint Inflammation in Rheumatoid Arthritis. N Engl J Med 2001;344:907-16. doi: 10.1056/NEJM200103223441207.

[4] Wakefield RJ, D'Agostino MA, Naredo E, et al. After treat-totarget: can a targeted ultrasound initiative improve RA outcomes? Ann Rheum Dis 2012;71:799-803. doi: 10.1136/annrheumdis2011-201048

[5] Maini R, St Clair, Breedveld F, et al. Infliximab (chimeric antitumour necrosis factor alpha monoclonal antibody) versus placebo in rheumatoid arthritis patients receiving concomitant methotrexate: a randomised phase III trial. ATTRACT Study Group. Lancet (London, England) 1999;354:1932-9. doi: 10.1016/ s0140-6736(99)05246-0.

[6] Bathon JM, Martin RW, Fleischmann RM, et al. A Comparison of Etanercept and Methotrexate in Patients with Early Rheumatoid Arthritis. N Engl J Med 2000;343:1586-93. doi: 10.1056/NEJM 200011303432201

[7] Criswell LA, Lum RF, Turner KN, et al. The influence of genetic variation in the HLA-DRB1 and LTA-TNF regions on the response to treatment of early rheumatoid arthritis with methotrexate or etanercept. Arthritis Rheum 2004. doi: 10.1002/art.20469.

[8] Sandrine G, Nathalie B, Julien B, et al. Influence of -308 A/G polymorphism in the tumor necrosis factor $\alpha$ gene on etanercept treatment in rheumatoid arthritis. Arthritis Care Res 2007. doi: 10.1002/art.23092.

[9] Ongaro A, De Mattei M, Pellati A, et al. Can tumor necrosis factor receptor II gene 676T $>\mathrm{G}$ polymorphism predict the response grading to anti-TNF $\alpha$ therapy in rheumatoid arthritis? Rheumatol Int 2008;28:901-8. doi: 10.1007/s00296-008-0552-5.

[10] Julià A, Erra A, Palacio C, et al. An eight-gene blood expression profile predicts the response to infliximab in rheumatoid arthritis. PLoS One 2009;4:e7556. doi: 10.1371/journal.pone.0007556.

[11] Schotte H, Schlüter B, Drynda S, et al. Interleukin 10 promoter microsatellite polymorphisms are associated with response to long term treatment with etanercept in patients with rheumatoid arthritis. Ann Rheum Dis 2005;64:575-81. doi: 10.1136/ $\operatorname{ard} .2004 .027672$.

[12] Tolusso B, Pietrapertosa D, Morelli A, et al. IL-1B and IL-1RN gene polymorphisms in rheumatoid arthritis: relationship with protein plasma levels and response to therapy. Pharmacogenomics 2006;7:683-95. doi: 10.2217/14622416.7.5.683.

[13] Padyukov L, Lampa J, Heimbürger M, et al. Genetic markers for the efficacy of tumour necrosis factor blocking therapy in rheumatoid arthritis. Ann Rheum Dis 2003;62:526-9. doi: 10.1136/ $\operatorname{ard} .62 .6 .526$.

[14] Nishikawa R, Nagai H, Bito T, et al. Genetic prediction of the effectiveness of biologics for psoriasis treatment. J Dermatol 2016; 43:1273-7. doi: 10.1111/1346-8138.13412.

[15] Honne K, Hallgrímsdóttir I, Wu C, et al. A longitudinal genomewide association study of anti-tumor necrosis factor response among Japanese patients with rheumatoid arthritis. Arthritis Res Ther 2016;18:12. doi: 10.1186/s13075-016-0920-6.

[16] Cui J, Stahl EA, Saevarsdottir S, et al. Genome-wide association study and gene expression analysis identifies CD84 as a predictor of response to etanercept therapy in rheumatoid arthritis. PLoS
Genet 2013;9:e1003394. doi: 10.1371/journal.pgen.1003394.

[17] Tanino M, Matoba R, Nakamura S, et al. Prediction of efficacy of anti-TNF biologic agent, infliximab, for rheumatoid arthritis patients using a comprehensive transcriptome analysis of white blood cells. Biochem Biophys Res Commun 2009;387:261-5. doi: 10.1016/j.bbrc.2009.06.149.

[18] Fabre S, Dupuy AM, Dossat N, et al. Protein biochip array technology for cytokine profiling predicts etanercept responsiveness in rheumatoid arthritis. Clin Exp Immunol 2008;153:188-95. doi: 10.1111/j.1365-2249.2008.03691.x.

[19] Nishimura K, Sugiyama D, Kogata Y, et al. Meta-analysis: Diagnostic Accuracy of Anti-Cyclic Citrullinated Peptide Antibody and Rheumatoid Factor for Rheumatoid Arthritis. Ann Intern Med 2007;146:797. doi: 10.7326/0003-4819-146-11-200706050-00008.

[20] Bosè F, Raeli L, Garutti C, et al. Dual role of anti-TNF therapy: enhancement of TCR-mediated $\mathrm{T}$ cell activation in peripheral blood and inhibition of inflammation in target tissues. Clin Immunol 2011;139:164-76. doi: 10.1016/j.clim.2011.01.015.

[21] Schmidt CS, Liu J, Zhang T, et al. Enhanced B cell expansion, survival, and humoral responses by targeting death receptor 6 . J Exp Med 2003;197:51-62.

[22] Liu J, Na S, Glasebrook A, et al. Enhanced CD4+ T cell proliferation and Th2 cytokine production in DR6-deficient mice. Immunity 2001;15:23-34.

[23] Kasof GM, Lu JJ, Liu D, et al. Tumor necrosis factor- $\alpha$ induces the expression of DR6, a member of the TNF receptor family, through activation of NF- $\kappa$ B. Oncogene 2001;20:7965-75. doi: 10.1038/sj.onc. 1204985.

[24] Okada Y, Terao C, Ikari K, et al. Meta-analysis identifies nine new loci associated with rheumatoid arthritis in the Japanese population. Nat Genet 2012;44:511-6. doi: 10.1038/ng.2231.

[25] Hock BD, O'Donnell JL, Taylor K, et al. Levels of the soluble forms of CD80, CD86, and CD83 are elevated in the synovial fluid of rheumatoid arthritis patients. Tissue Antigens 2006;67:5760. doi: 10.1111/j.1399-0039.2005.00524.x.

[26] Kristensen A-M, Stengaard-Pedersen K, Hetland ML, et al. Expression of soluble CD83 in plasma from early-stage rheumatoid arthritis patients is not modified by anti-TNF- $\alpha$ therapy. Cytokine 2017;96:1-7. doi: 10.1016/j.cyto.2017.02.017.

[27] Hamann J, Wishaupt JO, Van Lier RAW, et al. Expression of the activation antigen CD97 and its ligand CD55 in rheumatoid synovial tissue. Arthritis Rheum 1999;42:650-8. doi: 10.1002/ 1529-0131(199904)42:4<650:AID-ANR7>3.0.CO;2-S.

[28] Wakai K, Hamajima N, Okada R, et al. Profile of Participants and Genotype Distributions of 108 Polymorphisms in a CrossSectional Study of Associations of Genotypes With Lifestyle and Clinical Factors: A Project in the Japan Multi-Institutional Collaborative Cohort (J-MICC) Study. J Epidemiol 2011;21:223-35. doi: 10.2188/jea.JE20100139.

[29] Hashimoto M, Watanabe M, Uematsu Y, et al. Relationships of alcohol dehydrogenase 1B (ADH1B) and aldehyde dehydrogenase 2 (ALDH2) genotypes with alcohol sensitivity, drinking behavior and problem drinking in Japanese older men. Environ Health Prev Med 2016. doi: 10.1007/s12199-016-0507-5. 